Case Report

\title{
Cytological and histopathological diagnosis of a multifocal Rosai dorfman disease with involvement of Pinna-A rare case report
}

\author{
Sheela K.M1', Ruby Elizabeth ${ }^{2}$, Divya $\mathbf{R}^{3}$ \\ ${ }^{1}$ Dr. Sheela K.M, Associate Professor (CAP), Government Medical College, Trivandrum, ${ }^{2}$ Dr. Ruby Elizabeth Elias, \\ Associate Professor (CAP), Government Medical College, Trivandrum, ${ }^{3}$ Dr. Divya R, Junior Resident, Government \\ Medical College, Trivandrum, Kerala, India. \\ Corresponding Author: Dr. Sheela K.M, Associate Professor (CAP), Government Medical College, Trivandrum, \\ Kerala, India. E-mail: sheelakm51@gmail.com
}

\begin{abstract}
Rosai-Dorfman disease (RDD) is a rare lymph proliferative disorder with nodal and extranodal involvements. We are presenting this case of Rosai dorfman disease with presentation in pinna of ear and with previous history of Rosai dorfman disease at multiple sites including lymph node. Cytological and histological examination of the submandibular lymph nodes and skin biopsy demonstrated evidences of RDD.
\end{abstract}

Keywords: Rosai Dorfman Disease, Sinus Histiocytosis, Extranodal, Multifocal

\section{Introduction}

Rosai dorfman disease was first described by Rosai and Dorfman as sinus histiocytosis with massive lymphadenopathy in the year 1969 [1]. RDD can affect various head and neck sites, including the nasal cavity, paranasal sinuses and nasopharynx. Concomitant cervical node involvement may or may not be present $[2,3]$. It is an idiopathic, nodal-based histiocytic proliferative disorder that usually resolves spontaneously $[4,5,6]$. About $43 \%$ of cases occur in extra-nodal disease, which can be widespread and most frequently involves the respiratory tract, paranasal sinuses, visceral organs, skin, bones, central nervous system, genitourinary tract and orbit [7]. In the absence of lymph node involvement, RDD limited to the skin is called as cutaneous Rosai-Dorfman disease (CRDD). To date, very few cases of CRDD involving the ear have been reported [8] [9] [10]. The diagnosis of Rosaidorfman disease is made on the basis of clinical presentation, histopathological examination and immunohistochemical studies.

\section{Case Report}

We are presenting a case of 16-year old female a resident of Marthandam in Kanyakumari district who is a known case of Rosaidorfman disease of cervical lymph node and nasopharynx, now presented with swelling right external ear since 2 months in ENT OPD. Swelling was aspirated and submitted to our cytology department for cytological diagnosis. On physical examination swelling measured $1 \times 0.8 \times 0.5 \mathrm{~cm}$, and it was soft, nontender, beaded in appearance and was located on the border of right pinna. She had no significant medical and family history General examination were within normal limits. Patient had a history of swelling nasopharynx and cervical lymph node one year back, which was aspirated and then excised later and was sent for cytological study and histopatholigical examination respectively. It was reported as Rosaidorfman disease both in cytological study and histopathological examination.

The aspirate from pinna was sent to us after fixing in isopropyl alcohol. The smear was then pap stained and examined under microscopy. The smear was cellular with inflammatory cells composed of large histiocytes with intact neutrophil and plasma cells within the cytoplasm of histiocytes (figure1). Also noted are multinucleated giant cell, lymphocyte, histiocytes and neutrophil (figure 2). We received two specimens one excised specimen from right external ear and other right external auditory canal mass. Grossly both were tiny grey white mucosal fragments one measuring $0.7 \mathrm{x} 0.5 \mathrm{x} 05 \mathrm{~cm}$ and other aggregate measuring $0.8 \times 0.5 \times 0.5 \mathrm{~cm}$ respectively. The specimens were excised and send to our department of

Manuscript received: $14^{\text {th }}$ November 2018

Reviewed: $24^{\text {th }}$ November 2018

Author Corrected: $30^{\text {th }}$ November 2018

Accepted for Publication: $6^{\text {th }}$ December 2018 


\section{Case Report}

pathology for histopathological examination after fixing in $10 \%$ neutral buffered formalin. On microscopic examination, section showed tissue lined by stratified squamous epithelium, subepithelium showed dense inflammatory infiltrate composed of lymphocytes, plasma cell, foamy histiocytes and neutrophils. Also noted were large histiocytes with pale eosinophilic cytoplasm vesicular nucleus, demonstrating emperipolesis. These cells were positive for S100 and negative for CD1a which ruled out Langerhans cell histiocytosis.

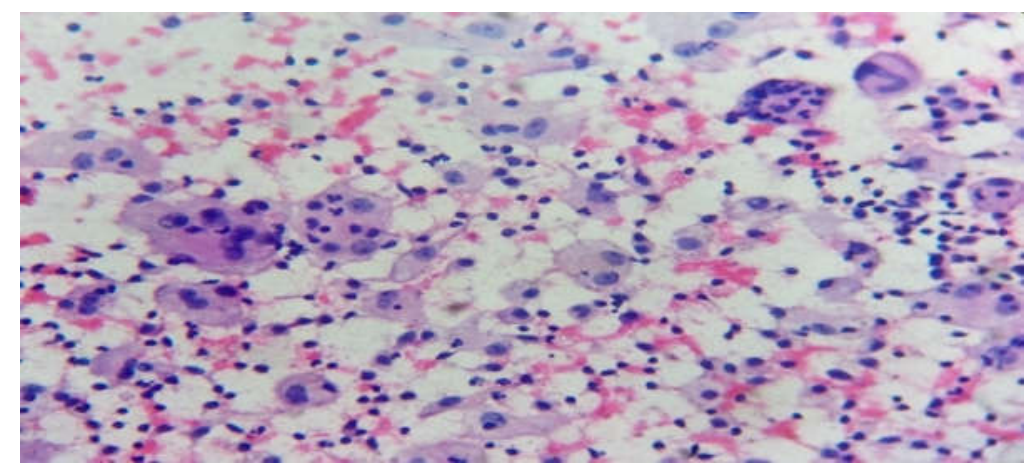

Figure-1: Pap stained smear showing emperipolesis with neutrophils and plasma cells in the cytoplasm of large histiocytes

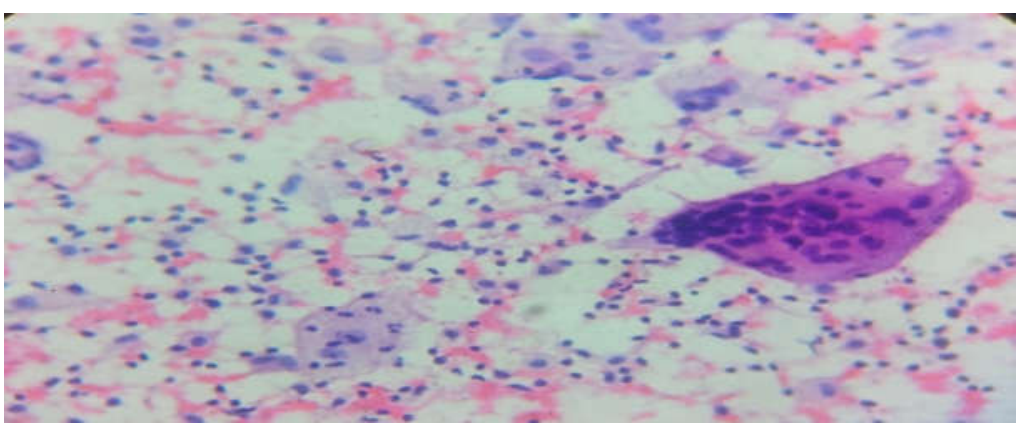

Figure-2: Showing emeperipolesis and multinucleated giant cells along with other inflammatory cells

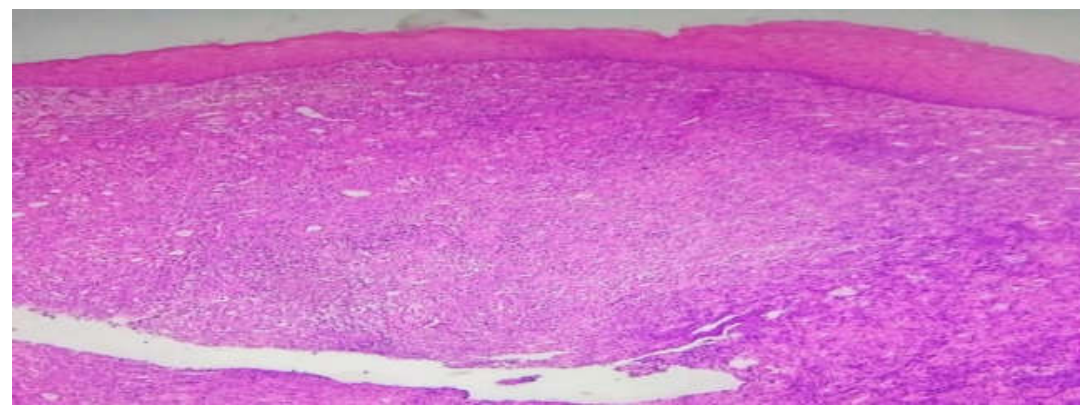

Figure-3: Showing stratified squamous epithelium and sub epithelium showing dense inflammation

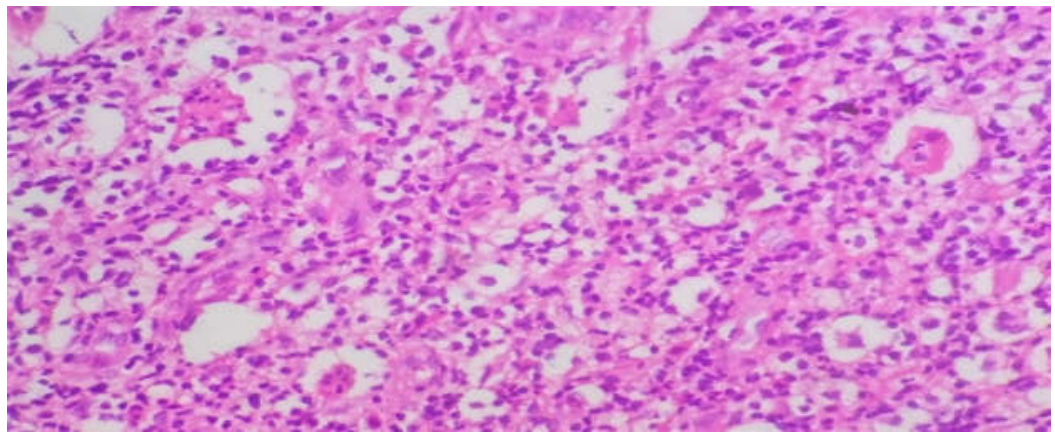

Figure-4: Showing inflammatory cells along with many hsitiocytes demonstrating emperipolesis 


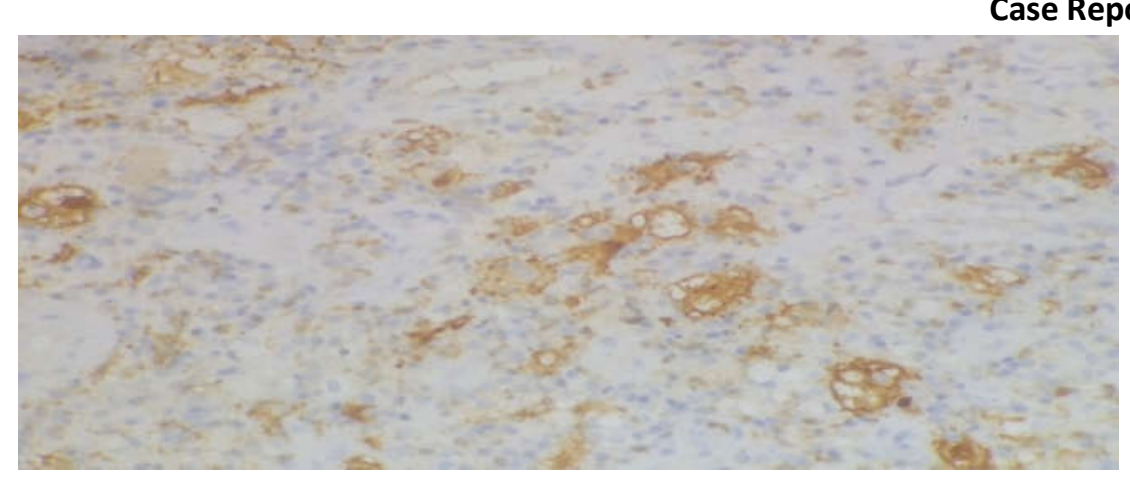

Figure-5: Demonstrating S100 positivity in rosaidorfman disease by histiocytes

\section{Discussion}

Emperipolesis is derived from the Greek (em - inside; peri - around; polemai - wander about) and was defined as "The active penetration of one cell by another which remains intact." It differs from phagocytosis in that an engulfed cell exists temporarily within another cell and with an intact normal structure while in phagocytosis, the engulfed cell is destroyed by the protective action of lysosomal enzymes. The cells involved in Emperipolesis are mainly histiocytes and megakaryocytes but can also be seen in association with tumour cells and Reed Sternberg cells. It is a hallmark feature of Rosai - Dorfman disease (RDD) but can also be part of other malignant conditions such as hematolymphoid disorders (Hodgkin's disease, leukaemia, acute and chronic myeloid leukaemia, Non Hodgkin's lymphoma, myeloproliferative disorders, myelodysplastic syndrome) and Non haematological malignancies (multiple myeloma, nueroblastoma, Rhabdomyosarcoma) [11].

From review of literature, only 5 cases of CRDD involving the ear have been reported $[8,9,10]$. In each of those cases, the ear was the only location on the skin involved. Cervical lymph nodes are typically involved, leading to painless bilateral cervical lymphadenopathy. Associated findings often include fever, leukocytosis, elevated erythrocyte sedimentation rate, and polyclonal hypergammaglobulinemia.

The classic form of the RDD typically presents in young adults with lymphadenopathy in the neck. Approximately $20-40 \%$ of patients experience disease remission, sometimes spontaneous. Extranodal RDD has a higher tendency for a chronic relapsing course and affects older individuals.

The cause of RDD is unknown; but the source have been postulated as autoimmune diseases and viruses such as human herpes virus 6, Epstein-Barr virus, parvovirus B19, lymphoma, Langerhans cell disease, autoimmune lymphoproliferative syndrome-1, joint disease, asthma, juvenile-onset diabetes, HIV, and red blood cell auto antibodies [11,12]. The pathogenesis involves recruitment of marrow monocytes from the peripheral blood into lymph node sinuses or extra nodal sites with subsequent transformation into the immunophenotypically distinct RDD histiocytes [13]. Histiocytes with abundant pale, vacuolated, or eosinophilic cytoplasm are typically seen within a field of lymphocytes and plasma cells [14]. Emperipolesis, the process by which histiocytes phagocytize intact lymphocytes or plasma cells, is characteristic of but is not pathognomonic for $\mathrm{RD}[6,12]$. These histiocytes can release cytokines, such as tumor necrosis factor- $\alpha$, which result in fever and other systemic symptoms [13].

RDD histiocytes share a common monocytemacrophage lineage with other S-100 immunostainpositive antigen presenting cells such as Langerhans cell histiocytosis (LCH). In contrast to $\mathrm{LCH}, \mathrm{CD} 1 \mathrm{a}$ is negative in RDD [13] [15]. In addition, RDD shows CD68 positivity and factor XIII a negativity. Extranodal RDD sites often show more fibrosis, fewer histiocytes, and less emperipolesis. Thus, a more thorough evaluation of the sample and additional immunohistochemical stains may be needed to confirm the diagnosis [16]. The cytological findings in sinus histiocytosis with massive lymphadenopathy (RosaiDorfman disease) were described by Van Heerde and others [17]. In this condition, smears contain many large histiocytes showing lymphophagocytosis.

Although there is no specific imaging characteristics that allow differentiation of lymphadenopathy in RosaiDorfman disease from other disease processes, massive painless bilateral cervical lymph node enlargement, particularly when occurring in children and adolescents, should promote consideration of Rosai-Dorfman disease in the differential diagnosis [18]. Of extranodal sites affected, the skin is the most common, followed by the paranasal sinuses and nasal cavity, subcutaneous tissue, orbit, eyelids, and bone Multiple additional sites of 


\section{Case Report}

extranodal involvement in the head and neck have been described, including the salivary glands, oral cavity, pharynx, tonsils, and trachea [12] [19]. Okada et al. [20] reported a case of tracheal involvement confined to the mucosa, without involvement of the tracheal cartilage. Additional lesions in the carina and left main bronchus were also present, as well as enlarged mediastinal lymph nodes [20]. Juskevicius and Finley [21] described a case of parotid gland involvement without significant lymphadenopathy in a 48-year-old patient with systemic lupus erythematosus. The orbit, globe, and eyelid are also common sites of involvement in Rosai-Dorfman disease, occurring in $7-10 \%$ of patients.

CNS involvement with Rosai-Dorfman disease often has no associated lymphadenopathy (up to $70 \%$ of patients), and it commonly occurs in adult men, usually in the fourth decade; however, it has also been reported in children [22]. Common locations are the parasellar, cavernous sinus, petroclival, and parafalcine regions; the cerebello-pontine angle; and posterior fossa. Primary osseous involvement with Rosai- Dorfman disease is rare but has been reported to involve the maxilla [23], as in one of our patients, as well as the sphenoid bone and the frontal bone [22].

The nonspecific appearance of cutaneous lesions can lead to a broad differential diagnosis, including other histiocytoses, sarcoidosis, tuberculosis, leishmaniasis, syphilis, and other infectious and infiltrative etiologies. The diagnosis of RDD was only made after performing immunohisto-chemistry on the tissue biopsy. Ear involvement is of particular significance because when present, it can help narrow the clinical and histologic differential diagnosis to include infiltrative and inflammatory processes involving the ear such as leprosy, relapsing polychondritis, perichondritis, pseudocyst of the auricle, and lymphoproliferative disorders. Numerous treatment options have been used for patients with CRDD including radiotherapy, corticosteroids (topical and systemic), chemotherapy, acyclovir, surgical excision, antibiotics, cryotherapy, interferon, dapsone, and retinoids [24].

\section{Conclusion}

The diagnosis of RDD should be considered in any atypical chronic inflammatory lesion with a histiocytic component involving multiple organ systems. The disease often follows a benign clinical course, with poorer prognosis associated with a heavy nodal burden and systemic involvement. The ideal treatment of CRDD has yet to be determined. We propose the development of a national registry of patients for future meta-analyses to help gain a better understanding of the disease etiology, clinical features, and future treatment options.

Findings: Nil; Conflict of Interest: None initiated Permission from IRB: Yes

\section{References}

1. Foucar E, Rosai J, Dorfman R. Sinus histiocytosis with massive lymphadenopathy (Rosai-Dorfman disease): review of the entity. Semin Diagn Pathol. 1990 Feb;7(1):19-73.

2. Wenig BM, Abbondanzo SL, Childers EL, et al. Extranodal sinus histiocytosis with massive lymphadenopathy (Rosai-Dorfman disease) of the head and neck. Hum Pathol 1993; 24:483-492.

3. Foucar E, Rosai J, Dorfman RF. Sinus histiocytosis with massive lymphadenopathy. An analysis of 14 deaths occurring in a patient registry. Cancer. 1984 Nov 1; 54 (9):1834-40.

4. Rosai J, Dorfman RF. Sinus histiocytosis with massive lymphadenopathy. A newly recognized benign clinicopathological entity. Arch Pathol. 1969 Jan;87(1): 63-70.

5. Rosai J, Dorfman RF. Sinus histiocytosis with massive lymphadenopathy: a pseudolymphomatous benign disorder. Analysis of 34 cases. Cancer. 1972 Nov; 30 (5):1174-88.

6. Foucar E, Rosai J, Dorfman R. Sinus histiocytosis with massive lymphadenopathy (Rosai-Dorfman disease): review of the entity. Semin Diagn Pathol. 1990 Feb;7(1):19-73.

7. Lim R, Wittram C, Ferry JA, et al. FDG PET of Rosai-Dorfman disease of the thymus. AJR Am J Roentgenol. 2004 Feb;182 (2):514. DOI:10.2214/ajr. 182. 2. 1820514

8.Tsang WY, Chan JK, Ho WK, et al. Extranodal Rosai-Dorfman disease: an uncommon cause of persistent nodule in the ear. J Laryngol Otol. 1992; 106:249-251.

9. Ahsan SF, Madgy DN, Poulik J. Otolaryngologic manifestations of Rosai-Dorfman Disease. Int J Pediatr Otorhinolaryngol. 2001 Jul 2;59(3):221-7.

10. Oo KK, Pang YT, Thamboo TP. Bilateral cauliflower ear deformity: an unusual presentation of cutaneous Rosai-Dorfman disease. Plast Reconstr Surg. 2004 Mar;113(3):967-9. 


\section{Case Report}

11. Maia RC, de Meis E, Romano S, et al. RosaiDorfman disease: a report of eight cases in a tertiary care center and a review of the literature. Braz $\mathrm{J}$ Med Biol Res. 2015 Jan;48(1):6-12. Epub 2014 Oct 10.

12. McAlister WH, Herman T, Dehner LP. Sinus histiocytosis with massive lymphadenopathy (RosaiDorfman disease). Pediatr Radiol. 1990;20(6):425-32.

13. Foss HD, Herbst H, Araujo I, et al. Monokine expression in Langerhans' cell histiocytosis and sinus histiocytosis with massive lymphadenopathy (RosaiDorfman disease) J Pathol. 1996 May; 179(1):60-5. DOI:10.1002/ (SICI)1096-9896 (199605) 179: $1<60:$ : AID-PATH533>3.0.CO;2-F

14. Swerdlow SH, Campo E, Harris NL, et al., eds. WHO classification of tumours of haematopoieticand lymphoid tissues, vol. 2, 4th ed. Lyon, France: IARC Press, 2008

15. Wang $\mathrm{KH}$, Cheng $\mathrm{CJ}$, $\mathrm{Hu} \mathrm{CH}$, et al. Coexistence of localized Langerhans cell histiocytosis and cutaneous Rosai-Dorfman disease. Br J Dermatol. 2002 Oct;147 (4):770-4

16. Mar WA, Yu JH, Knuttinen MG, et al. RosaiDorfman Disease: Manifestations Outside of the Head and Neck. AJR Am J Roentgenol. 2017 Apr; 208 (4): 721-732. doi: 10.2214/AJR.15.15504. Epub 2017 Jan 31.

17. Kumar B, Karki S, Paudyal P. Diagnosis of sinus histiocytosis with massive lymphadenopathy (RosaiDorfman disease) by fine needle aspiration cytology. Diagn Cytopathol. 2008 Oct;36(10):691-5. doi: 10.1002 /dc. 20904
18. Ahuja AT, Ying M. Sonographic evaluation of cervical lymph nodes. AJR Am J Roentgenol. 2005 May; 184(5):1691-9. DOI:10.2214/ajr.184.5.01841691.

19.Yiğitbaşi O, Güney E, Unal A. Sinus histiocytosis with massive lymphadenopathy. Otolaryngol Head Neck Surg 1999; 121:507.

20.Okada K, Lee MO, Hitomi S, et al. Sinus histiocytosis with massive lymphadenopathy and tracheobronchial lesions: CT and MR findings. J Comput Assist Tomogr 1988; 12:1039-1040.

21. Juskevicius R, Finley JL. Rosai-Dorfman disease of the parotid gland: cytologic and histopathologic findings with immunohistochemical correlation. Arch Pathol Lab Med. 2001 Oct;125 (10): 1348-50. DOI:10.1043/0003-9985(2001)125<1348:RDDOTP> 2.0.CO;2.

22. Rodriguez-Galindo C, Helton K, Sánchez N, Rieman M, Jeng M, Wang W. Extranodal RosaiDorfman disease in children. J Pediatr Hematol Oncol 2004; 26:19-24.

23. Shemen L, D'Anton M, Klijian A. Rosai-Dorfman disease involving the premaxilla. Ann Otol Rhinol Laryngol. 1991 Oct;100(10):845-51 DOI:10.1177/000 348949110001011.

24. Safdar A, Gillenwater AM, Jones DM, et al. RosaiDorfman disease misdiagnosed as active tuberculosis. Leuk Lymphoma. 2006 Jul; 47 (7):1441-2. DOI:10.1080/10428190600625521.

\section{How to cite this article?}

Sheela K.M, Ruby Elizabeth, Divya R. Cytological and histopathological diagnosis of a multifocal Rosai dorfman disease with involvement of Pinna- A rare case report. Trop J Path Micro 2018;4(8):551-555. doi:10.17511/jopm.2018.i08.01. 